\title{
La sabatierite, un nouveau séléniure de cuivre et de thallium
}

Zdenek Johan, Milan Kvacek, Paul Picot

\section{Citer ce document / Cite this document :}

Johan Zdenek, Kvacek Milan, Picot Paul. La sabatierite, un nouveau séléniure de cuivre et de thallium. In: Bulletin de Minéralogie, volume 101, 5-6, 1978. pp. 557-560;

doi : https://doi.org/10.3406/bulmi.1978.7227

https://www.persee.fr/doc/bulmi_0180-9210_1978_num_101_5_7227

Fichier pdf généré le 15/01/2021 


\begin{abstract}
Sabatierite, $\mathrm{Cu}_{6} \mathrm{TISe}_{4}$, is orthorhombic (indexing of powder pattern) with $\mathrm{a}=3.986 ; \mathrm{b}=5.624 ; \mathrm{c}=$ $9.778 \AA ; Z=1$; the calculated specific gravity is $6.78 \mathrm{~g} / \mathrm{cm} 3$. The strongest lines in the powder pattern are : 3.987 (5), 3.089 (10), 2.706 (7), 2.525 (5), 2.445 (6), 1.991 (7), 1.847 (6), 1-673 (5) A. Average composition from microprobe analyses : Cu 42.71 ; T1 22.44 ; Se 34.57 , total $99.72 \%$, giving a formula Cu6.06TI0.99Se3.95. In reflected light it is bluish gray in colour with slightly cream tint. Reflectivity values : 33.2-31.0 (420) ; 32.2-29.5 (500) ; 30.6-27.9 (540) ; 27.2-25.3 (600) ; 21.6-21.3 (700 nm). Strongly anisotropic with undulatory extinction. Microhardness (Vickers) : $54 \pm 7 \mathrm{~kg} / \mathrm{mm} 2$. Found in Bukov ore deposit, Moravia, Czechoslovakia, associated with crookesite and berzelianite. Extremely rare. The name is for Germain Sabatier.
\end{abstract}

\title{
Résumé
}

La sabatierite, $\mathrm{Cu}_{6} \mathrm{TISe}_{4}$, est orthorhombique (indexation du diagramme de poudre), avec a $=3,986, \mathrm{~b}$ $=5,624, c=9,778 \AA, Z=1$; la densité calculée est égale à $6,78 \mathrm{~g} / \mathrm{cm} 3$. Raies les plus intenses du diagramme de poudre : 3,987 (5), 3,089 (10), 2,706 (7), 2,525 (5), 2,445 (6), 1,991 (7), 1,847 (6), 1,673 (5) Å. Les analyses à la microsonde électronique ont donné la composition moyenne suivante : $\mathrm{Cu}$ 42,71 ; 1122,44 i Se 34,57, total 99,72 \%, conduisant à la formule $\mathrm{Cu}_{6,06} \mathrm{Tl}_{0,99} \mathrm{Se}_{3,95}$. En lumière réfléchie, la sabatierite est gris bleu avec une légère teinte crème. Pouvoir réflecteur : 33,2-31,0 (420); 32,2-29-5 (500) ; 30,6-27,9 (540) ; 27,2-25,3 (600) ; 21,6-21,3 (700 nm). Fortement anisotrope ; extinction roulante. Microdureté (Vickers) : $54 \pm 7 \mathrm{~kg} / \mathrm{mm} 2$. Trouvée dans le gisement de Bukov, Moravie, Tchécoslovaquie, associée à la crookesite et à la berzelianite. Très rare. Nommée en I'honneur de Germain Sabatier. 
PUGA, E. (1970). - Tres variedades de cloritoide en Sierra Nevada (Cordilleras Beticas, Espana). Cuad. Geol. Univ. Granada, $1,65-70$.

IRAO, T. R. (1970). - The occurrence of ottrelite along the thrust zone in the Pakhals of Yellandlapad area, Andrha Pradesh, India. Geol. Mag., IO6, 452-456.

Rauw, H. (de) (I9II). - Note sur la salmite, le rutile et la tourmaline d'Ottré. Ann. Soc. géol. Belgique, 38, B 209-B 2I 4.
Renard, A. et Vallée Poussin, C. (de la) (I879). Note sur l'ottrélite. Ann. Soc. géol. Belgique, 6, 5 I-68. TARTE, P. (1958). - IRecherches sur les fréquences de déformation $\mathrm{OH}$. I : Spectre infrarouge des sels basiques de cuivre. Spectrochim. Acta, I3, ro7-119.

Wilson, A. D. (1960). - The micro-determination of ferrous iron in silicate minerals by a volumetric and a colorimetric method. The Analyst, 85, no roI6, $823-827$.

\title{
La sabatierite, un nouveau séléniure de cuivre et de thallium
}

\author{
par Zdenek JOHAN (1), Milan KVAČEK ( $\left.{ }^{2}\right)$ et Paul PICOT $\left({ }^{3}\right)$.
}

\begin{abstract}
Résumé. - La sabatierite, $\mathrm{Cu}_{6} \mathrm{TlSe}_{4}$, est orthorhombique (indexation du diagramme de poudre), avec $a=3,986, b=5,624, c=9,778 \AA, Z=\mathrm{I}$; la densité calculée est égale à $6,78 \mathrm{~g} / \mathrm{cm}^{3}$. IRaies les plus intenses du diagramme de poudre : 3,987 (5), 3,089 (10), 2,706 (7), 2,525 (5), 2,445 (6), r,99r (7), I,847 (6), r,673 (5) $\AA$. Les analyses à la microsonde électronique ont donné la composition moyenne suivante : $\mathrm{Cu} 42,7 \mathrm{I} ; \mathrm{Tl} 22,44$; Se 34,57, total $99,72 \%$, conduisant à la formule $\mathrm{Cu}_{6,06} \mathrm{Tl}_{0,99} \mathrm{Se}_{3,95}$. En lumière réfléchie, la sabatierite est gris bleu avec une légère teinte crème. Pouvoir réflecteur : 33,2-3I,O (420) ; $32,2-29-5$ (500) ; 30,6-27,9 (540) ; 27,2-25,3 (600); 2I,6-2I,3 (700 nm). Fortement anisotrope ; extinction roulante. Microdureté (Vickers) : $54 \neq 7 \mathrm{~kg} / \mathrm{mm}^{2}$. Trouvée dans le gisement de Bukov, Moravie, Tchécoslovaquie, associée à la crookesite et à la berzelianite. Très rare. Nommée en l'honneur de Germain Sabatier.
\end{abstract}

Mots clés : sabatierite, nouveau minéral, séléniure de Tl et Cu, crookesite.

Sabatierite, a new copper thallium selenide.

Abstract. - Sabatierite, $\mathrm{Cu}_{6}{ } \mathrm{TISe}_{4}$, is orthorhombic (indexing of powder pattern) with $a=3.986 ; b=$ $5.624 ; c=9.778 \AA ; Z=\mathrm{I}$; the calculated specific gravity is $6.78 \mathrm{~g} / \mathrm{cm}^{3}$. The strongest lines in the powder pattern are : 3.987 (5), 3.089 (10), 2.706 (7), 2.525 (5), 2.445 (6), r.99 ( 7), r.847 (6), r.673 (5) A. Average composition from microprobe analyses : $\mathrm{Cu} 42.7 \mathrm{I} ; \mathrm{Tl} 22.44$; Se 34.57 , total $99.72 \%$, giving a formula $\mathrm{Cu}_{6.08} \mathrm{Tl}_{0.99} \mathrm{Se}_{3.95}$. In reflected light it is bluish gray in colour with slightly cream tint. Reflectivity values : $33.2-3$ I.O (420);32.2-29.5 (500); 30.6-27.9 (540); 27.2-25.3 (600); 2 I.6-2 I.3 (700 nm). Strongly anisotropic with undulatory extinction. Microhardness (Vickers) : $54 \pm 7 \mathrm{~kg} / \mathrm{mm}^{2}$. Found in Bukov ore deposit, Moravia, Czechoslovakia, associated with crookesite and berzelianite. Extremely rare. The name is for Germain Sabatier.

Key words : sabatierite, new mineral, $\mathrm{Cu}$ and $\mathrm{Tl}$ selenide, crookesite.

\section{INTRODUCTION.}

Plusieurs structures filoniennes, minéralisées en pechblende et renfermant une riche paragenèse de séléniures et de sulfures, ont été découvertes il y a une dizaine d'années, dans le bouclier du Massif de Bohême.

De nombreuses publications ont été consacrées à l'étude des minéraux provenant de ces gisements parmi lesquels il faut citer plusieurs espèces nouvelles,

(I) G. I. S. I3. R. G. M.-C. N. R. S., Centre de Recherche sur la Synthèse et la Chimie des Minéraux, IA, rue de la Férollerie, 45045 Orléans Cedex.

(2) Ústav nerostných surovin, Kutná Hora, Tchécoslovaquie.

(3) B. R. G. M., Service géologique national, I3. P. 6009, 45018 Orléans Cedex. telles que : bukovite (Johan et Kvaček, I97x b), hakite (Johan et Kvaček, I97I $a$ ), fischesserite (Johan et al., I97 I $b$ ), permingeatite (Johan et al., I97 I $a$ ), krutaite (Johan it al., I972), bellidoite (De Montreuil, I975), petrovicite (Johan et al., I976). Une description détaillée des paragenèses a été donnée par Kvaček (I965) et (1973). Il convient de souligner que du point de vue géochimique, ces minéralisations sont extrêmement complexes car plus qu'une dizaine d'éléments s'y trouvent minéralogiquement exprimés : $\mathrm{Ag}, \mathrm{Au}, \mathrm{Bi}, \mathrm{Co}, \mathrm{Cu}, \mathrm{Fe}, \mathrm{Hg}, \mathrm{Ni}, \mathrm{Pb}, \mathrm{Pd}, \mathrm{Sb}, \mathrm{Te}, \mathrm{Tl}$.

Le gisement de Bukov, faisant partie du district minier de Rožná et situé dans la zone morave du Mo!danubien, s'est révélé particulièrement riche en thallium. En effet, on y trouve de la crookesite (Kvaček, I966) formant parfois des accumulations dans la berzelianite massive et de la bukovite, $\mathrm{Cu}_{3} \mathrm{TlFe}_{2} \mathrm{Se}_{4}$, 
découverte dans ce gisement (Johan et Kvaček, I97 I b). Très rarement, la crookesite est accompagnée d'un autre séléniure de cuivre et de thallium dont la description fait l'objet de cette note.

Nous proposons de nommer ce minéral sabatierite, en l'honneur de Germain Sabatier, directeur de recherche au C. N. R.S., ancien directeur du Centre de Recherche sur la Synthèse et la Chimie des minéraux à Orléans, France ( $\left.{ }^{1}\right)$.

\section{Propriétés physigues et ol'tigues.}

En lumière réfléchie, à l'air, la sabatierite a un pouvoir réflecteur nettement moins élevé que la crookesite, à peine plus fort que celui de la berzelianite (fig. I). Par rapport à la berzelianite, sa couleur est

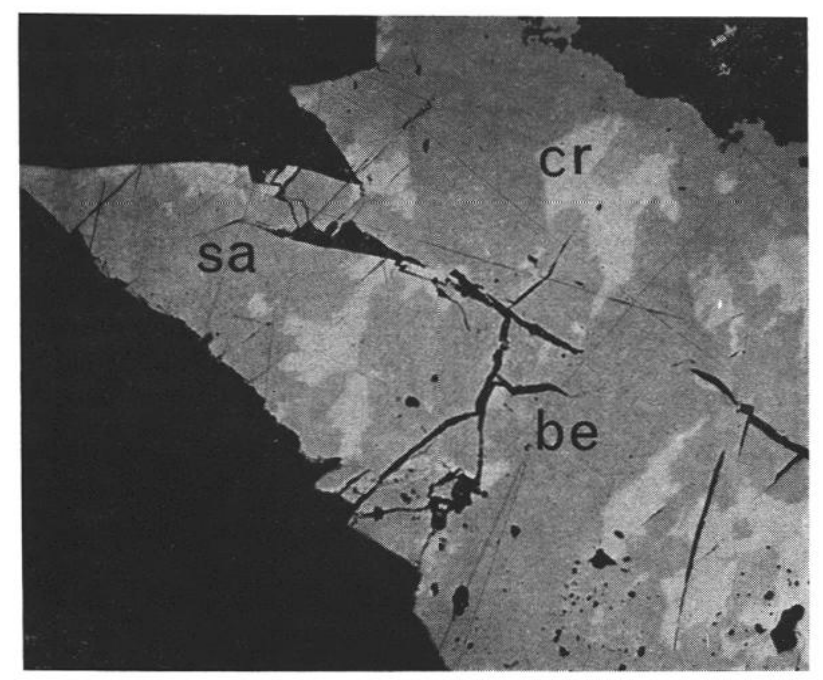

$a$

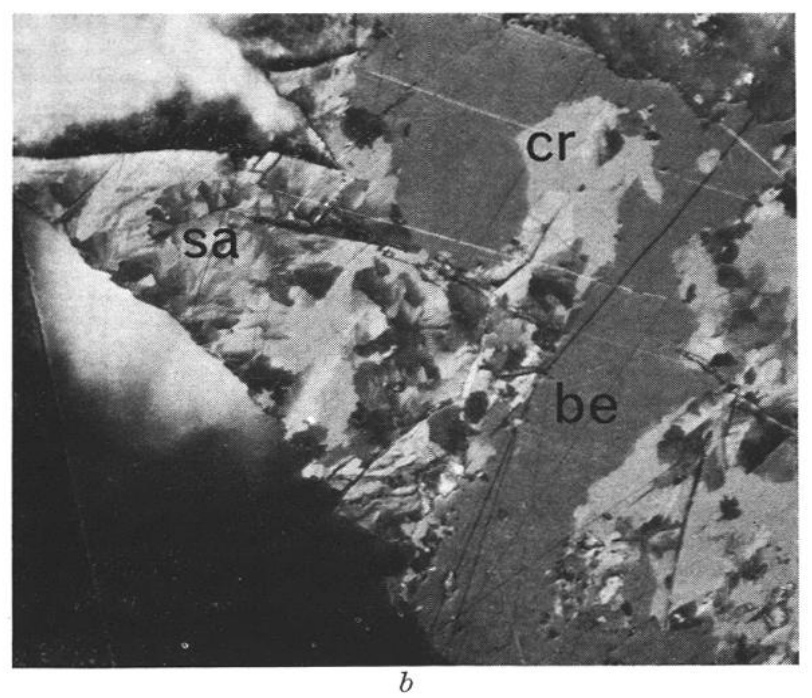

lis. I. - Sabatierite (sa) en agrégats radiaux remplaçant la crookesite (cr) incluse dans la berzelianite (be). Tous ces minéraux sont englobés dans une gangue carbonatée (calcite).

a) section polie, lumière réfléchie non analysée, immersion, 200

b) section polie, nicols croisés, immersion, $220 \because$.

(I) Ia description de la sabatierize a été approuvée par la Conmission internationale des nouveaux minéraux de l'I. M. A. par I7 voix contre o, vote du 2 I.10.1976. Nowveau nom approuvé par I r vix contre o, vote du 24.03 .1978 . gris bleuâtre avec une légère teinte crème. La différence des pouvoirs réflecteurs de ces deux minéraux et de leurs couleurs est si faible qu'en lumière réfléchie non analysće, la présence des plages de sabatierite incluses dans la berzelianite peut facilement échapper à l'observation. A l'air, le pléochroïsme du nouveau minéral est faible mais perceptible; dans l'huile, il devient net, allant de brun clair à bleu clair, moins franchement bleu que la berzelianite.

L'anisotropie est forte avec des effets chromatiques colorés : gris-bleu clair à jaune-brun foncé entre nicols complètement croisés. En les décroisant partiellement, on voit apparaître les couleurs brun-rouge foncé à gris bleuâtre. L'examen entre nicols croisés révèle que la sabatierite se présente en agrégats polycristallins à structure radiée et à extinction roulante (fig. I). Cette structure nous a obligés à effectuer des mesures de pouvoir réflecteur sur les plages polycristallines. De ce fait, $R_{\min }$ et $R_{\max }$ (tabl. I) repré-

TABLEAU I.

\section{Pouvoirs réflecteurs de la sabatierite.}

Monochromateur à prisme, photomultiplicateur à cathode $\mathrm{S}_{20}$, objectif $44 / 0,65$, étalon $\mathrm{SiC}$ (C. O. M.), compensation d'obliquité par $\left(\frac{R_{1}-1}{2}-R_{I}\right)$

\begin{tabular}{|l|l|l|}
\hline$\lambda(\mathrm{nm})$ & $R_{\max }(\%)$ & $R_{\min }(\%)$ \\
\hline 420 & 33.2 & 31.0 \\
440 & 34.3 & 30.9 \\
460 & 33.8 & 30.6 \\
480 & 33.0 & 30.1 \\
500 & 32.2 & 29.5 \\
520 & 31.5 & 28.7 \\
540 & 30.6 & 27.9 \\
560 & 29.6 & 27.0 \\
580 & 28.4 & 26.1 \\
600 & 27.2 & 25.3 \\
620 & 26.0 & 24.5 \\
640 & 24.8 & 23.8 \\
660 & 23.8 & 23.0 \\
680 & 22.7 & 22.2 \\
700 & 21.6 & 21.3 \\
\hline
\end{tabular}

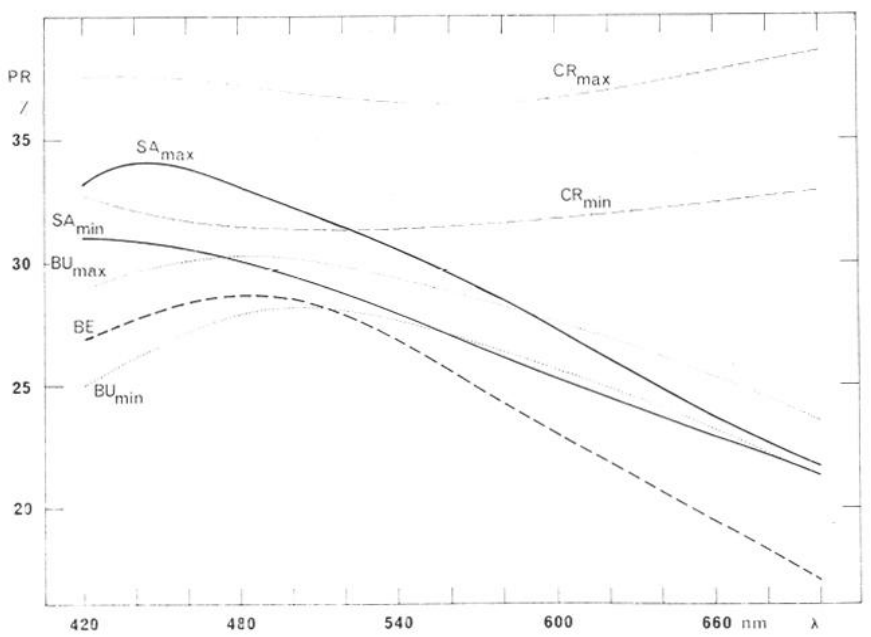

Fig. 2. - Comparaison des courbes de pouvoir réflecteur de : sabatierite (SA), berzelianite (BE), bukovite (BU) et crookesite (CR), provenant du gisement de Bukov, Moravie, Tchécoslovaquie. 
sentent les valeurs extrêmes observées sur les plages d'orientation quelconque. Pour toutes les longueurs d'onde, le pouvoir réflecteur de la sabatierite est supérieur à celui de la berzelianite, en se situant entre le pouvoir réflecteur de cette dernière et de la crookesite dont la courbe a cependant une allure différente (fig. 2). La comparaison avec la bukovite montre que son pouvoir réflecteur est plus faible entre 420 et $580 \mathrm{~nm}$, devenant supérieur pour les longueurs d'onde plus grandes.

La microdureté Vickers (moyenne de 5 mesures) est de $54 \pm 7 \mathrm{~kg} / \mathrm{mm}^{2}$ ( $\left.\mathrm{P}=\mathrm{I} 5 \mathrm{~g}\right)$, proche de celle de la bukovite $\left(62 \mathrm{~kg} / \mathrm{mm}^{2}\right)$. Les empreintes sont toujours fortement déformées. Par suite de sa rareté, la densité de la sabatierite n'a pas pu être mesurée; la densité calculée est égale à $6.78 \mathrm{~g} / \mathrm{cm}^{3}$.

\section{Composition CHIMIQUE.}

Hormis $\mathrm{Cu}, \mathrm{Tl}$ et $\mathrm{Se}$, aucun autre élément n'a été mis en évidence dans la sabatierite par analyse qualitative à la microsonde électronique. Une trentaine d'analyses de différentes plages du nouveau minéral ont été réalisées à la microsonde automatisée Came-

\section{ÉTUde CRISTALlographiQUe.}

En dépit de plusieurs essais, nous n'avons pas réussi à prélever, sur section polie, un éclat convenable permettant d'effectuer une étude sur monocristal. L'aspect général du diagramme de poudre, présentant des analogies avec le diagramme de poudrc de la rohaite (Karup-Møller, sous presse), ainsi que les propriétés optiques et la forme des agrégats de la sabatierite nous ont fait penser que sa structure pourrait être comparable à celle de la bukovite, caractérisée par les couches tétraédriques ${ }_{\infty}^{2}\{\mathrm{Cu}$ i $\mathrm{Se}\}$ et le thallium en position interfoliaire (Zemann, I973). Cette hypothèse semblait se confirmer, car le diagramme de poudre de la sabatierite a pu être indexé avec une maille quadratique ayant $a=3,986, c==2 \times 9,792 \AA, V=3$ II $\AA^{3}$. On remarque aussitôt que cette maille est commesurable suivant $a$ avec les mailles de : bukovite (Johan et Kvaček, I97 I b), thalcusite (Kovalenker et al., I976) et rohaite (Karup-Møller, sous presse). Cependant, son volume est incompatible avec la formule $\mathrm{Cu}_{6} \mathrm{TlSe}_{4}$ puisqu'il conduit à une densité calculée trop élevée $\left(9,65 \mathrm{~g} / \mathrm{cm}^{3}\right)$.

\section{TABLEAU II.}

\section{Analyses chimiques de la sabatierite et de la crookesite de Bukov (Tchécoslovaquie).}

I. Résultat d'analyse à la microsonde électronique (J. Breton et C. Gilles, analystes, B. R. G. M., Service géologique national, Orléans); témoins : $\mathrm{TlAsS}_{2}, \mathrm{Cu}$ et Se métaux.

2. Nombre d'atomes par unité formulaire calculé sur la base d'un total de II atomes pour la sabatierite et de I2 atomes pour la crookesite.

3. Compositions théoriques pour $\mathrm{Cu}_{6} \mathrm{TlSe}_{4}$ (sabatierite) et $\mathrm{Cu}_{7} \mathrm{TlSe}_{4}$ (crookesite).

\begin{tabular}{|c|c|c|c|c|c|c|c|c|c|c|c|}
\hline \multicolumn{12}{|c|}{ SABATIERITE } \\
\hline & \multicolumn{2}{|c|}{1} & \multicolumn{2}{|c|}{2} & \multicolumn{2}{|c|}{3} & \multicolumn{2}{|c|}{4} & \multicolumn{2}{|c|}{5} & \multirow[b]{2}{*}{3} \\
\hline & 1 & 2 & 1 & 2 & 1 & 2 & 1 & 2 & 1 & 2 & \\
\hline Cu & 42.87 & 6.06 & 42.35 & 6.05 & 42.33 & 6.03 & 43.35 & 6.10 & 42.67 & 6.06 & 42.29 \\
\hline וז & 22.30 & 0.98 & 22.78 & 1.01 & 22.25 & 0.99 & 22.35 & 0.98 & 22.51 & 0.99 & 22.67 \\
\hline Se & 34.75 & 3.95 & 34.27 & 3.94 & 34.71 & 3.98 & 34.67 & 3.92 & 34.46 & 3.94 & 35.04 \\
\hline$\Sigma$ & 99.92 & & 99.40 & & 99.29 & & 100.37 & & 99.64 & & 100.00 \\
\hline \multicolumn{12}{|c|}{ CROOKESITE } \\
\hline & \multicolumn{2}{|c|}{1} & \multicolumn{2}{|c|}{2} & \multicolumn{2}{|c|}{3} & \multicolumn{2}{|c|}{4} & \multicolumn{2}{|c|}{5} & \\
\hline & 1 & 2 & 1 & 2 & 1 & 2 & 1 & 2 & 1 & 2 & 3 \\
\hline $\mathrm{Cu}$ & 46.97 & 7.08 & 46.51 & 7.04 & 47.28 & 7.08 & 47.19 & 7.12 & 46.47 & 7.04 & 46.09 \\
\hline וז & 19.42 & 0.91 & 20.79 & 0.98 & 21.29 & 0.99 & 20.67 & 0.97 & 22.24 & 1.05 & 21.18 \\
\hline Se & 33.00 & 4.01 & 32.67 & 3.98 & 32.63 & 3.93 & 32.16 & 3.91 & 32.10 & 3.91 & 32.73 \\
\hline$\Sigma$ & 99.39 & & 99.97 & & 101.20 & & 100.02 & & 100.81 & & 100.00 \\
\hline
\end{tabular}

bax, en utilisant $\mathrm{TIAsS}_{2}$, Cu et Se métaux comme témoins. Cinq analyses les plus caractéristiques sont données au tableau II. Leurs résultats conduisent à des formules cristallochimiques très proches de la formule idéale $\mathrm{Cu}_{6} \mathrm{TlSe}_{4}$.

La crookesite associée à la sabatierite a été également analysée. Les résultats montrent (tabl. II) que son rapport $\mathrm{Cu} / \mathrm{Tl}$ est plus élevé, étant égal à 7 ; la formule de la crookesite doit par conséquent s'écrire. $\mathrm{Cu}_{7} \mathrm{TlSe}_{4}$. La maille quadratique proposée par Earley (I950) contient $2\left\{\mathrm{Cu}_{7} \mathrm{TlSe}_{4}\right\}$. La densité calculée est de $7,49 \mathrm{~g} / \mathrm{cm}^{3}$. Ainsi, bien que très distincte par ses propriétés optiques, la crookesite a une formule chimique proche de celle de la sabatierite.
Ceci nous a amenés à supposer que la maille de la sabatierite est en réalité orthorhombique, pseudoquadratique, avec $b \sim a \cdot \sqrt{2}$. En poursuivant ce raisonnement, nous avons abouti, après l'affinement des paramètres à partir du diagramme de poudre, au résultat suivant :

$$
\begin{aligned}
& a=3,986 \text { (4) } A \\
& b=5,624(4) \\
& c=9,778(8)
\end{aligned}
$$

$a: b: c=0,708_{7}: \mathrm{I}: \mathrm{I}, 738_{6}, Z=\mathrm{I}, \mathrm{V}=2 \mathrm{Ig}, 2(5) A^{3}$.

Le diagramme de poudre indexé (tabl. III) permet de voir que la maille est du type $\mathrm{P}$; aucune extinction systématique ne peut en être déduite avec certitude. 
TABLEAC ILI.

\section{Diagramme de poudre de la sabatierite.}

$\mathrm{Cu} / \mathrm{Ni}$, chambre de $240 \mathrm{~mm}$ de circonférence, intensités relatives de I à Io.

\begin{tabular}{|c|c|c|c|}
\hline I & $d_{\text {mes }}$ & ${ }^{d} \mathrm{calc}$ & hkl \\
\hline 5 & $3.987 \AA$ & $3.987 \AA$ & 100 \\
\hline 2 & 3.705 & 3.696 & 101 \\
\hline 10 & 3.089 & 3.089 & 102 \\
\hline 2 . & 2.818 & 2.813 & 020 \\
\hline 7 & 2.706 & 2.702 & 021 \\
\hline 5 & 2.525 & 2.523 & 103 \\
\hline 6 & 2.445 & 2.444 & 004 \\
\hline 4 & 2.129 & 2.129 & 023 \\
\hline 3 & 2.087 & 2.084 & 104 \\
\hline 7 & 1.991 & 1.993 & 200 \\
\hline 6 & 1.847 & 1.845 & 202 \\
\hline 1 & 1.786 & & \\
\hline \multirow[t]{2}{*}{5} & 1.673 & 1.674 & 124 \\
\hline & & 1.671 & 131 \\
\hline \multirow[t]{2}{*}{3} & 1.609 & 1.605 & 025 \\
\hline & & 1.603 & 132 \\
\hline 3 & 1.565 & 1.565 & 016 \\
\hline 3 & 1.406 & 1.406 & 040 \\
\hline 2 & 1.266 & 1.261 & 206 \\
\hline
\end{tabular}

Une raie d'intensité très faible avec $d=\mathrm{I}, 786 \AA$ reste non indexée. Nous l'attribuons à la présence, en traces, d'un minéral du groupe de la tyrrellite-trüstedtite, observé en inclusions dans la berzelianite et la crookesite associées à la sabatierite.

La densité calculée avec $Z=\mathrm{I}$ et la formule idéale de la sabatierite est de $6,78 \mathrm{~g} / \mathrm{cm}^{3}$, comparable aux densités des composés chimiquement proches, tels que $\mathrm{CuTlSe}_{2}\left(6,97 \mathrm{~g} / \mathrm{cm}^{3}\right.$, Cambi et Elli, Ig68) ou $\mathrm{Cu}_{1,8} \mathrm{Se}$ $\left(6,70 \mathrm{~g} / \mathrm{cm}^{3}\right)$. Nous pouvons donc considérer, à défaut d'une détermination directe, la maille indiquée comme très probable, soulignant toutefois la nécessité d'une confirmation par l'étude sur monocristal.

\section{CONDITIONS DE GISEMENT.}

Les filons à gangue carbonatée du gisement de Bukov recoupent une série de : gneiss à biotite et amphibole, amphibolites, quartzites et gneiss silicocalciques, appartenant au Moldanubien. D'après Kvaček (r973), cinq épisodes minéralisateurs peuvent être distingués, dont un correspondant au dépôt de pechblende accompagnée d'oxydes de fer et de vanadium (hématite, goethite, montroseite) et de séléniures. Cet épisode est précédé par la cristallisation des carbonates et suivi d'un développement de sulfures de cuivre (chalcopyrite, bornite, chalcocite) et de la pyrite.

La paragenèse des séléniures est particulièrement riche comprenant : berzelianite, bukovite, clausthalite, crookesite, eskebornite, eucaïrite, hakite, klockmannite, tyrrellite et umangite. Alors que la bukovite a été surtout observée dans les accumulations locales de l'umangite, la sabatierite se trouve intimement associée à la crookesite et à la berzelianite.

I.es observations en section polie mettent en évidence un remplacement métasomatique de la crookesite par la sabatierite (fig. I). Ceci laisse entrevoir la possibilité de formation de cette dernière par une réaction entre la crookesite préexistante et une solution hydrothermale contenant $\mathrm{Tl}$ et Se.

La sabatierite est un minéral très rare qui n'a été jusqu'à présent observé que dans deux sections polies. L'échantillon type est déposé dans la collection de l'École supérieure des mines de Paris.

\section{REMERCIEMENTS.}

Nous tenons à remercier M. le Dr E. Makovicky de l'Université de Copenhague pour les suggestions et les critiques qui nous ont considérablement aidés, et M. le $D^{r}$ S. Karup-Møller de la même Université qui nous a aimablement communiqué les données en cours de publication sur la rohaite.

\section{Reçu le I9 juillet 1978 .}

Accepté le Io octobre 1978 .

\section{BIBLIOGRAPHIE}

Cambi, L. et Elli, M. (I968). - Seleniuri semplici e complessi : sintesi idrotermale da ossidi di metalli o metalloidi e selenio. Chim. Industr. Ital., 5o, 95-105.

De Montreuil, L. A. (I975). - Bellidoite : a new copper selenide. Econ. Geol., 7o, 384-387.

EARLEY, J. W. (1950). - Description and synthesis of the selenide minerals. Amer. Mineral., 35, 337-364.

Johax, Z. et KVAČEK, M. (I97 I $a$ ). - La hakite, un nouveau minćral du groupc de la tétraédrite. Bull. Soc. $f r$. Minéral. Cristallogr., 94, 45-48.

JoHAN, Z. et KVAČEK, M. (197I $b$ ). - La bukovite, $\mathrm{Cu}_{3+x} \mathrm{Tl}_{2} \mathrm{FeSe}_{4-x}$, une nouvelle espèce minérale. Bull. Soc. fr. Minéral. Cristallogr., 94, 529-533.

Johan, Z., Picot, P., PiERrot, IR. et KVAČeK, M. (I97I a). - I a permingeatite, $\mathrm{Cu}_{3} \mathrm{SbSe}_{1}$, un nouveau minéral du groupe de la luzonite. Bull. Soc. fr. Minéral. Cristallogr., 94, I62-165.

Johan, Z., Picot, P., Pierrot, R. et KVaček, M. $($ I97I $b)$. - La fischesserite, $\mathrm{Ag}_{3} \mathrm{AuSe}_{2}$, premier séléniure d'or, isotype de la petzite. Bull. Soc. $f r$. Minéral. Cristallogr., 94, 381-384.

Johan, Z., Picot, P., Pierrot, R. et Kvaček, M. (I972). - La krutaïte, $\mathrm{CuSe}_{2}$, un nouveau minéral du groupe de la pyrite. Büll. Soc. fr. Minéral. Cristal$\log r ., 95,475^{-48} \mathrm{I}$.
Johax, Z., KVAČeK, M. et PICOT, P. (1976). - La petrovicite, $\mathrm{Cu}_{3} \mathrm{HgPbBiSe}_{5}$, un nouveau minéral. Bull. Soc. fr. Minéral. Cristallogr., 99, $3^{10-313 .}$

Karup-Møller, S. (sous presse). - Rohaite, a new thallium sulphide from the Ilimaussaq alkaline intrusion in South Greenland.

Kovalenker, V. A., Laputina, I. P., Evstignefva, T. L. et Izortioo, V. M. (r976). - Thalcusite, $\mathrm{Cu}_{3-x} \mathrm{Tl}_{2} \mathrm{Fe}_{1+x} \mathrm{~S}_{4}$, un nouveau sulfure de thallium provenant des minerais de cuivre et de nickel du gisement de Talnakh (en russe). Zap. Vses. Min. Obsch., 105, 202-206.

KVAČ́K, M. (I965). - Beitrag zur Geochemie der Selenidvererzung im Ceskomoravská Vrchovina Gebiet-CSSR. Sborn. 1. geoch. Konference, Ostrava, 393-402.

KVAČ́K, M. (I966).--Occurrence of crookesite at Bukov. Zprávy Vlast. Úst. Olomouc, I26, I-2.

Kvaček, M. (1973). - Selenides from the uranium deposits of Western Moravia, Czechoslovakia. Part I. Berzelianite, umangite, eskebornite. Acta Univ. Carolinae, Geologica, Rost vol., 23-36.

Zemain, J. (1973). - Der Strukturtyp des Bukovits. Anz. math.-naturw. Klasse, Öster. Akad. I'iss., No. 10, I26-I 28 . 